\title{
Understanding Issues Around Use of Oral Pre- Exposure Prophylaxis Among Female Sex Workers in India.
}

Seema Sahay ( $\sim$ ssahay@nariindia.org )

National AIDS Research Institute

Archana Verma

National AIDS Research Institute

Suhas Shewale

National AIDS Research Institute

Sampada Dipak Bangar

National AIDS Research Institute

Athokpam Bijeshkumar

National AIDS Research Institute

Mubashir Angolkar

Jawaharlal Nehru Medical College

Thilakavathi Subramanian

National Institute of Epidemiology

Nomita Chandhiok

Indian Council of Medical Research

\section{Research article}

Keywords: FSW, oral PrEP, India, qualitative research, violence, stigma, fear of side-effects, alcohol use, adherence, reproductive health

Posted Date: May 7th, 2021

DOl: https://doi.org/10.21203/rs.3.rs-493162/v1

License: (c) (i) This work is licensed under a Creative Commons Attribution 4.0 International License.

Read Full License 


\section{Abstract}

\section{Background}

Empowering female sex workers (FSWs) through women controlled HIV prevention option has been in focus globally. FSWs are important target for oral PrEP (Pre Exposure Prophylaxis). A multi-centric qualitative study was conducted to explore the FSWs' willingness to use oral PrEP in India.

\section{Methods}

Seventy three qualitative interviews and 02 focus group discussions were conducted at 3 high HIV prevalent states in India during 2013-14. Study explored issues around willingness to use oral PrEP. The study was approved by the respective institutional ethics committee of the study sites. Thematic analysis using grounded theory approach was used to analyze the data in N-VIVO version 8.0.

\section{Results}

Thematic analysis showed events of forced condom-less sex. FSW believed that oral PrEP could provide independence, financial gains, and privacy and therefore hoped to use it as an alternative to male condom. However, any impact on physical/ aesthetic attributes and reproductive system were not acceptable and could become a barrier. Provider initiated oral PrEP was not preferred. Providers voiced safety monitoring concerns. Adherence emerged as a challenge because of: 1) alcohol use; 2) taking PrEP tablet each day being too mundane; 3 ) Stigma because Oral PrEP is ARV based. Alcohol use and dread of repetitive dose brings forth the need for long acting oral PrEP.

\section{Conclusion}

Oral PrEP is acceptable among FSWs; it should be rolled out alongside strong messages on STI protection and PrEP as compliment to condoms. PrEP roll out requires educating communities about HIV treatment versus prevention. Long-acting oral PrEP could address both 'mundane' and alcoholism and sustain adherence.

\section{Background}

The Indian epidemic is concentrated among vulnerable populations at high risk for HIV (1). The concentrated epidemics are driven by unprotected sex between sex workers and their clients, men having sex with men and by injecting drug use with contaminated injections (2). National AIDS Control Programme Phase-IV (2012-17) in India aimed to strengthen the response to the epidemic with key strategies of intensifying and consolidating prevention services with a focus on high-risk groups (HRGs) and vulnerable populations (3). Female Sex Workers (FSWs) is the population that has shown response to the Combination HIV prevention program that included behavioral change communication, barrier strategies (condom promotion), and structural programs (4) as evident from the declining trend in HIV prevalence between the year 2007 (5.06\%) and 2011 (5). Hence, FSWs might respond well to new HIV 
prevention options also. Therefore, empowering FSWs through women controlled HIV prevention options to reduce their risk of HIV infection has been a major focus of prevention efforts globally. Several clinical trials reported protective effects of biomedical interventions in the form of gel and oral formulations of pre-exposure prophylaxis (PrEP) but some trials were also stopped because they lacked efficacy (6-8). Subsequent studies showed that lower efficacy was related to suboptimal adherence to the $\operatorname{PrEP}(9,10)$. Therefore, the dose-response relationship between efficacy and adherence to PrEP has received critical focus among researchers because of the subjective world view of adherence taking precedence over objective world view. An enhanced understanding of social and behavioral influences on PrEP use has been recommended $(11,12)$.

UNAIDS and the World Health Organization recommend PrEP as an additional prevention choice for people at substantial risk of HIV exposure and who are ready to have regular HIV testing. However, a gap between efficacy and effectiveness is foreseen especially as clinical trial researchers observed adherence as a major challenge.

Adherence to preventive strategies is challenging because there is often no direct effect such as the reduction in any kind of 'symptoms' or getting a feeling of wellness. Acceptance and adherence are shaped within the socio-cultural, psychological and programmatic context in any geographical setting. Despite the strong biological effectiveness of oral PrEP owing to strong adherence, FSWs face many structural challenges to PrEP uptake and use (13). Social scientists have raised the need to assess the subjective world view and social meanings of PrEP. They have warned to take cognizance of the perception of safety, trust, and empowerment (14). A PrEP demonstration project was conducted among FSWs in Kolkata, India which has shown that there is a demand for PrEP among FSWs but sociostructural barriers influence PrEP uptake (15). Studies on needs and perception of FSWs about PrEP uptake and its delivery is a gap in India. According to Bandura (1986), the human function is influenced by one's characteristics; environment or context and behavior (16). To understand the basic human function of decision making to use a preventive product in environmental, behavioral and personal context; a qualitative study was undertaken to get an emic perspective for oral PrEP uptake among the FSWs in India. Parkin's theory of decision making process of problem definition; thought; judgment; decision; and action (17) was utilized to interpret the data.

\section{Methods}

\section{Study setting}

Based on the NACO's categorization of districts as per HIV prevalence, two districts in each state which were identified as 'category A' (> 1\% ANC prevalence in the district in any of the sites in the last 3 years) and 'category $B^{\prime}$ ( $<1 \%$ ANC prevalence in all the sites during last 3 years with $>5 \%$ prevalence in any HRG site) (3) were selected as study sites. Using this criterion, Pune (urban) and Satara (rural) districts in the state of Maharashtra; Belagavi (urban) and Hubli-Dharwad (rural) districts in the state of Karnataka; and Chennai (urban) and Vellore (rural) districts in the state of Tamil Nadu were selected as study sites. The 
participants were categorized into 3 components: 1) Potential PrEP Users: FSWs, 2) Key informants: Brothel owners/ keepers, and 3) PrEP Providers: Health care providers.

\section{Sampling and study population}

Purposive and convenience sampling techniques were used to recruit the participants and face to face interviews or group discussions were conducted. The participants were identified and recruited through Non-Governmental Organizations (NGOs), Community Based Organizations (CBOs) and key community gatekeepers such as tea stall owner/ worker, paan waala (the betel shop owner), small shop keepers and pimps. A total of 39 in-depth interviews (IDIs) were conducted with FSWs as follows: 1) Brothel Based Sex Workers (BBSWs) $(n=20)$, and 2) Street Based Sex Workers (SBSWs) $(n=19)$. Thirty-four key informant interviews (KIIs) were also conducted with six brothel keepers (BKs), one bisexual man and 27 health care providers/ program personnel. Two focus group discussions (FGDs) were conducted with a total of 14 FSWs at two urban sites of Pune and Chennai respectively. BBSWs and BKs were approached through NGOs/ CBOs only. SBSWs were approached either through NGOs or other SBSWs using the snowball technique. The participants were self-identified as BBSWs, SBSWs, and BKs. The HIV status of the participants was not explored in the study. The health care providers, included physicians, obstetric gynecologists which were identified from government and private health centers, counselors from ICTC and NGO/CBO, and representatives from District AIDS Prevention and Control Society (DAPCU). Interviews were conducted at a confidential and convenient place agreed on by the participant. Each interview took 40-90 minutes. Four FSWs refused to participate in the study.

To provide a background understanding of the subject, the participants received information on recent research findings of oral PrEP before conducting the interviews/ FGDs. A description of oral PrEP used as a preamble before the interview section about oral PrEP.

\section{Study tools \& data collection}

The interview guides for IDI and FGD focused on the perceived need for HIV prevention, attitudes and practices about family planning methods; knowledge and experiences of condom use for family planning and/ or prevention; knowledge, perception about STI and HIV/ AIDS, prevention methods, and usage modalities for oral PrEP. The study explored the opinion and expectations of the FSWs from oral PrEP, barriers and facilitators in using oral PrEP, and willingness to use oral PrEP. The guides were translated into local vernacular languages (Hindi, Marathi, Kannada, Tamil, and Telugu) by the study sites. The guides were pilot tested at all study sites and refined based on pilot findings.

All the interviews were conducted by trained master's level female social workers in the local language in Maharashtra and Tamil Nadu. Data from Karnataka was collected by a trained female social worker and MPH female students. Field notes were taken during the IDIs and FGDs. The data were audio-recorded and data collection was continued until theoretical saturation was reached. As fieldwork progressed, data was continuously analyzed using constant comparison and iterative thematic coding approach (18). The reimbursement of Rs. 150 was given to all study participants. 


\section{Data analysis}

The audio data was transcribed verbatim, translated into English and typed in Microsoft Word at the study sites. The processed translated electronic data were repeatedly reviewed by two researchers and the PI. Repeat interviews were requested from the sites in case of missing information or if there was a need for new information. The repeated sections underwent a similar process of data processing and finalized data was entered in qualitative software N-VIVO version 8.0. Attributes were tabulated in the software to quantify demographic variables of location, typology, age, and marital status. Data were coded to recognize similarities and differences using the constant comparison method (19). Firstly, the themes were identified deductively from the interview guide and subsequently inductively from the data. Following the iterative process of reading, final themes emerged using the grounded theory approach (20). Data were coded by two researchers SS and AV, first independently and then discussed together. The codes and the descriptors were shared with the site Pls for confirmation and their interpretations were included to preserve the local meanings. The coded data were analyzed which informed the next iteration of data collection until a strong theoretical understanding was attained and these are described as emerging themes.

\section{Ethics}

The study was approved by the Institutional Ethics Committees of all the 3 sites: the ICMR-National AIDS Research Institute (Pune, Maharashtra), the ICMR-National Institute of Epidemiology (Chennai, Tamil Nadu) and the Jawaharlal Nehru Medical College (Belagavi, Karnataka). Written informed consent was obtained from all the study participants for participation in the study and audio recording their responses before conducting the IDIs, KIIs, and FGDs.

\section{Results}

Of the total 59 FSW participants, 42 belonged to the urban setting while the rest belonged to the rural setting. The mean age of the FSWs in both urban and rural settings was similar ( 35.7 years, SD= 8.8). The data was analyzed thematically to capture willingness to use oral PrEP and understand the dynamics of the various factors that affect the usage, acceptance, and adherence of PrEP among a highrisk HIV group, the FSWs, in various parts of India.

All the FSWs from both the settings reported experiencing coercive sex which involuntarily brought out the risk of condom-less sex. Several other situations also led to condom-less sex. The main emerging theme in this study was 'condom-less sex which led to the theme of 'oral PrEP is a felt need'. The latter encompassed issues such as female-controlled options, an alternative to male condom, long-term protection, easy to use and economically viable option. As PrEP is not rolled out in India, respondents expressed the testing of PrEP before actual usage. Therefore, 'building confidence in product' was another emerging theme for acceptance. Influencers for PrEP acceptance and usage emerged as barriers and facilitators for PrEP usage among the FSW population. However, the reiterative readings helped in identifying the characteristics and issues that would lead to acceptance of oral PrEP under the theme 
'expected product characteristics' (Figure 1). This theme covered the issues of ease of use, ensuring privacy and positioning PrEP in a way to maintain confidentiality. Experiences of antiretroviral therapy and their job profile threw light on both acceptance and adherence issues. 'Adherence' was another theme in the study.

\section{\{lnsert Figure 1: Analytical framework for emerging needs for oral PrEP use among FSWs\}}

The major themes that emerged from the study were: 1) Condom-less sex, 2) Oral PrEP is a felt need, 3) Building confidence in the product, 4) Expected product characteristics, 5) Barriers to acceptance, and 6) Adherence to oral PrEP.

\section{Context of condom-less sex}

While explaining their work, FSWs shared about their vulnerabilities. Commenting on the lack of protection policy for their population, they narrated incidences of coercive sex and situations where they would end up having condom-less sex. FGD participants unanimously reported that like any other woman, they too faced resistance to condom use in regular partner settings. 'Suspicion'because of condom use; an emphatic 'no' to condom use by the partner; 'confidence on fidelity' - were the phrases used by FSWs to explain condom-less sex in certain types of relationships. FSWs stressed that livelihood needs itself brought vulnerability. An SBSW from Pune narrated the situation where being aware of the risk did not necessarily result in the practice of using a condom:

"Now I came to you for work [/maid work/] but that owner likes me. If the female owner went outside, then, he takes that maid servant to the bed [for sex]. Is there a condom [available at that time]?" [Urban

\section{SBSW, 01-10-13, Pune, MH]}

The universal nature of the risk was apparent at all the settings. Violence and getting caught in the exploiting situations where FSWs were forced to provide services to multiple people, were reported at all the sites in both rural and urban areas. An FGD participant from the same city reported:

"Some person [/client/] will call us and ask us to have sex in the nearby bushes. When we go, we see there, then there will be 6 to 7 people. It is very much difficult to handle the situation, and cannot use a condom with all of them!" [Urban FGD, FSW-4, Chennai, TN].

In rural Karnataka (Hubli district), FSWs talked of fear of getting killed if they resisted:

"What to do? We don't have any options. They [/clients/] come and tell that there is only one person, but they will be four and they will take [us] to the jungle and will do [sex] forcefully. If we go against them, they will kill us and will throw [us] there only" [Rural BBSW, 03-10-35, Hubli-Dharwad, KT].

Instead of didactic education, practical need for protection emerged. Need for protection was urgent because of fear even from law enforcers. A lodge-based FSW from Belagavi explained as follows: 
"Government should give us more facility [/safe space/] that also in free. We are poor. So sometimes police and 'don' [/local goons/] people come and take [us] forcefully [for sex] and put us in jail, and then they are asking for money and doing nonsense things [/having sex/] with us. Then madam [/ Brothel Keeper/] goes from here to bring us out from jail. We are in serious trouble in Belagavi". [Urban BBSW, 0310-35, Belagavi, KT]

FSWs did not use condom with their regular partner for fear of suspicion and violence when condom use was suggested:

"We cannot insist upon all [/types of partners/] to use condom. If we insist on a husband or partner to use a condom, then they will suspect us and violence might happen" [Urban FGD, FSW-2, Chennai, TN].

The emotional need of getting accepted by the regular partner led to condom-less sex even among the empowered peers. 'Trust' in a relationship turned out to be a barrier to condom use:

"My partner has seen my report. I don't have HIV [infection]. [He] has seen my report, and he sits [/has sex/] with me without a condom. What to do?" [Urban SBSW, 01-10-13, Pune, MH]

In the setting of stigma and societal facelessness, trust shown by her partner is a valued reflection of respect given by the partner which she would not want to lose at any cost. To reciprocate, she does away with condom use. An FSW in Chennai stated:

"I don't have a husband but I have one regular partner. And he has a lot of confidence in me. So due to this high confidence in me, I never use a condom with him" [Urban FGD, FSW-4, Chennai, TN]

\section{Oral PrEP is a felt need}

Despite being aware of risks and working in the fear of situations of coercive sex, FSWs did not refuse to go 'outside' with clients. Condom-less sex events seemed to be a common occurrence. Varying levels of risk brought out the need for FSW controlled prevention option. An FSW shared her need for oral PrEP as follows:

"Sometimes if we get caught in the custody of rowdies, they threaten us and ask us to have sex without using a condom. So because of fear, we have sex without a condom with them. To prevent HIV transmission it [/oral PrEP/] is needed, like earlier sister said that, at the time of alcohol consumption they do sex without a condom" [Urban FGD, FSW-1, Chennai, TN].

In the context of condom-less sex, oral PrEP as an alternative to male condom was a felt need of both rural and urban FSWs:

"Definitely, the new prevention product is very much necessary because the gents [/men/] refuse to use the condom during sex. This new prevention product will be very much necessary for people like us [/FSWs/]'[Rural BBSW, 06-10-44, Vellore, KN]. 
Oral PrEP was thought as addressing following issues: Pleasure; protection and financially beneficial at individual level of FSW profession.

Pleasure: Need for oral PrEP, especially as a female-controlled option, was acceptable to both the brothel $(42.7 \%)$ and street-based (57\%) sex workers. They reported facing challenges around male condom usage such as 'interference with pleasure' and 'client satisfaction'. A female key informant from an NGO emphasized upon the client's expectation of pleasure and how oral PrEP would satisfy that criteria:

"It [/oral PrEP/] is convenient to take! There is no question. People feel that there is no skin to skin touch in condom use. That issue doesn't come in that [/when one uses oral PrEP/]'[Urban Social worker, 01-6016, Pune, $M H]$.

FSWs in the FGD agreed as follows:

"Some people say that they feel dissatisfaction during sex [/with condom/]" [Urban FGD, FSW-5, Chennai, $\mathrm{TN}]$.

More Controlled Protection: Violence and forced sex were the social conditions in which an FSW lived. Within this social context, an FSW who is aware of the threats but her livelihood needs make her take a risk, depicts the multi-layered nature of her vulnerabilities. Transactional sex is 'hurried' which leads to condom tear. These situations give rise to the need for other prevention technologies:

"Some customers [/client/] will tear the condom and do [sex]. In such times, we should be careful. So this tablet [/PrEP/] will be useful in that condition'[Urban SBSW, 05-10-19, Chennai, TN].

Since client satisfaction is the major goal in the sex work profession, a health care provider (HCP) from a rural site emphasized PrEP as an empowerment option for FSWs.

"If it [/PrEP/] is to be taken regularly, target intervention people especially female sex worker can take it. Because they can use it when the male condom is not used. A female condom is not there [/available/]" [Rural DAPCU representative, 06-50-41, Vellore, KN].

Profitable: Condom-less sex being more in demand, FSWs participating in FGD had hoped for an alternative for the male condom. They examined its economic benefit along with its long term protection value:

"Some people are there if the clients give more money then, they have sex without using a condom" [Urban FGD, FSW-2, Chennai, TN].

She emphasized her need for long term protection which a tablet formulation would be able to give her along with non negotiable sex work with clients who do not want condom. The empowerment due to PrEP tablet reflected as follows: 
"...It [/Prevention option/] must come in the form of a tablet so that it will be in our body and we can do sex work without any problem. Eventually, we can earn more money and also we can live safely" [Urban FGD, FSW-2, Chennai, TN].

No one talked about pregnancy prevention or STI prevention.

\section{Building confidence in the product}

A high level of acceptance for hypothetical oral PrEP was expressed among FSW belonging to the urban areas of Chennai (10/13) and Pune (03/05). However, the need to be confident about the actual product prevailed:

"Now, without seeing how we can tell [about] those things? [/she laughs/] Without seeing, how can the things be known? As we saw [/used/] the condom and accepted... so will check the thing [/oral PrEP/] after it comes and then only will prefer'[Urban SBSW, 01-10-13, Pune, MH].

"It is very much difficult to take the tablet regularly. Before taking this tablet [/PrEP/] regularly we must 'know' about the tablet [/PrEP/]'[Urban SBSW, 05-10-77, Chennai, TN].

Participants felt they need to use a product to know the range of side effects it may cause and it might influence the sustained use of this prevention product:

"Only after using it, we can tell whether it has any problem. But it should not have side effects.' [Urban SBSW, 05-10-19, Chennai, TN].

In Chennai, participants were conscious of the need for the product to be tested first:

"Before launching the tablet, the tablet must be pre-tested. Then only people will come forward to buy and use" [Urban FGD, FSW-3, Chennai, TN].

\section{Expected product characteristics}

If FSWs were confident about the product and wanted to use it, they had suggestions for certain characteristics of the product which would facilitate optimal usage of the product. Following three facilitators emerged from the study: 1) Easy to use, 2) Ensuring privacy-formulation, 3) Positioning to maintain confidentiality

Easy to use. Among many of the challenges with condom usage, making men wear a male condom was reported to be the major challenge for FSWs. They found the process of wearing a condom far tedious which in their own words was 'not as simple as swallowing a tablet'.

"This tablet is for preventing HIV. So, it can be very well taken. We don't have a fear of it. Whoever wants, can use it. Men or women, whoever is going to this type of work [/sex work/], they can use it. It is good to use it as it prevents HIV'[Urban BBSW, 05-10-27, Chennai, TN]. 
Formulation to ensure privacy: FSWs wanted to protect their privacy; a client need not know if she was using any protection. This privacy also appeared to be a function of 'trust'. Without invading the 'trust' of the partner, the FSW would be able to use the pill to protect herself. Swallowing a pill would give her protection, privacy, and ease of use, altogether. According to them, since it would be a pill, it would be easy to use:

"Among all, tablets are best madam. Means it will be swallowed if that tablet is taken-means- no one will come to know" [Rural SBSW, 04-10-08, Dharwad, KN].

The 'oral tablet' allayed FSWs' fears about their confidentiality; they felt that it made them self-sufficient for protection; did not make them dependent on the health system:

"Tablet is the best. We don't have to go to a place for injection, we can buy and keep the tablets and use by ourselves. Even this has to be made available in NGOs, medical shops and hospitals" [Urban SBSW, 05-10-19, Chennai, TN].

There seemed to be no realization that oral PrEP might not be an 'over the counter' (OTC) drug and that there would be a need for monitoring by the HCP for any side effects after the initiation of oral PrEP. FSWs from Chennai during FGD pointed out their preference for CBOs or NGOs and not doctors for oral PrEP dispensing as they wanted to be given services 'with no questions asked'.

"Now the doctor asks many questions - how many customers I have attended? Do you have a habit of alcohol? How much money do you spend on that?" [Urban FGD, FSW-7, Chennai, TN]

"This may be the reason people are not coming to the hospitals because unwanted questions were being asked by the doctors... so this may be the reason that people hesitate to come to the hospital and ICTC centers'[FSW-2].

Positioning to maintain confidentiality: The theme 'Positioning to maintain confidentiality was derived from the narratives where FSWs felt that they would be able to access the tablet in privacy; where no one would know them and no one would understand the purpose. According to them, the positioning of the product as a medical product would be helpful:

"It will be good if it comes in medical products like a tablet, so that without anyone knowing [about it], we can have the tablet" [Rural BBSW, 06-10-44, Vellore, TN].

“...normally anyone goes to medical [/pharmacy/]" [Urban SBSW, 01-10-13, Pune, MH].

Unlike FSWs in an urban setting who did not want to go to a doctor to receive oral PrEP, FSWs in rural areas did not have any concern about the integration of oral PrEP delivery with ART centers or ICTCs. Their idea was to integrate CBOs/ NGOs with existing program structures:

"From the ART center, it should be promoted up to the outside people again... it is very necessary [to be dispensed], through an organization. From there also the women will get information" [Rural BK, 02-60-44, 


\section{Karad, $\mathrm{MH}]$.}

"I feel that... somewhere if it [/tablet/] will be kept in NGOs then will be ok. I do feel so" [Rural SBSW, 0210-42, Karad, MH].

From the implementer's point of view also, dispensing through village level functionaries was the preferred option:

"Availability is very important for the tablet. If it is available with village health nurses, Anganwadi workers [/village level workers/] it will be still good" [Rural DAPCU representative,06-50-41, Vellore, KT].

\section{Barriers to acceptance}

Many concerns about oral PrEP were expressed. Taking a tablet for prevention is a major issue. Following barriers to oral PrEP usage emerged: 1) Stigma of using HIV treatment product, 2) Fear of side effects.

Stigma of using HIV treatment (ART) product: FSWs were apprehensive about accessing oral PrEP tablets because they felt that it would be equated to seeking treatment for HIV by the community. They used phrases such as, 'becominginfamous [/defamed/]'; 'community would never believe [/that they are uninfected/]'. Despite the emerging need for oral PrEP, FSWs cautioned that oral PrEP might become stigmatizing. After all, the Oral PrEP is an 'ARV'. They were conscious of the fact that the community perceived oral PrEP tablets as a 'treatment medicine' for 'the HIV infected'. To differentiate between oral PrEP and ARV was too complex to discern for them and the community:

"No one will agree for that tablet [/oral PrEP/]. So women will be afraid of becoming infamous. =name of $F S W=$ as well as =name of another FSW= eats the same tablets! It means they both have got [/HIV/]. There the people [/client/] will say this who is [/point out/] having the same tablets. That tablet [/ARV/] has become too famous" [Urban SBSW, 01-10-13, Pune, MH].

A woman mimicked the voice of the community:

"This means the tablets cannot be taken without a reason. I don't have any disease, nor any risk. Why then I eat [/tablet/]?'[Urban Woman (Spouse of PLHIV), 01-20-23, Pune, MH]

Fear of side effects: In addition to the above described reasons for non-use of oral PrEP, fear of side effects was another major cited reason. Most of the BBSWs (77.3\%) talked about their concerns about side effects. However, SBSWs raised this concern in low numbers (5.6\%).

“... But for some people, the body will not accept the drug. Sometimes they may take the tablet and some time they may skip'[Urban BBSW, 05-10-27, Chennai, TN].

'Body heat, giddiness, ulcers, weakness and total rejection by the body' were some of the anticipated side effects of oral PrEP. Fear of side effect was also voiced as reasons for preferring condoms over oral PrEP. 
"I don't know whether people will use regularly. Those who like to use condom definitely they may have fear on using this tablet [/PrEP/] so there will be less chance of using this tablet [/PrEP/]'[Urban BBSW, 05-10-72, Chennai, TN].

FSWs professed fear towards the possibility of harm occurring to their reproductive parts. They were quite vocal about this issue and some narrated their experience from family planning.

"But I feel difficult to use regularly because earlier I used =mala-d= [/contraceptive pills/] that gives many problems like a bad smell in urine, lower abdomen pain and burning sensation" [Urban BBSW, 05-10-72, Chennai, TN].

Related to their profession, preserving the beauty and the reproductive part/s, were some of the major concerns. In both urban and rural sites, concern existed among the FSWs that these attributes might get compromised if they took oral PrEP:

"People will take regularly to prevent diseases. This tablet we can take but there should not be any problem in the uterus of the women" [Rural BBSW, 06-10-44, Vellore, TN].

"Only if it is not having side effects, we can use it regularly. Beauty is a must to do sex work. This tablet should not spoil it" [Urban SBSW, 05-10-19, Chennai, TN].

\section{Perceived barriers to adherence}

Despite the proven efficacy of oral PrEP, the effectiveness depends on the adherence. The daily dose was a challenge:

"FSW people... Taking the tablet daily is impossible for them. Other products may be used by others" [Urban BBSW, 05-10-30, Chennai, TN].

Three sub-themes emerged that cover the perceived barriers to adherence to oral PrEP: 1) Alcoholism, 2) Psychological barriers, 3) Typological barriers. These barriers to adherence are discussed as follows:

Alcoholism: Alcohol consumption is a compulsion of her profession and subsequently, it becomes her need. Hence a vicious cycle of compulsion and alcoholism appears to be the lifestyle under which FSWs showed apprehension for initiating or adherence to oral PrEP tablets. According to an FSW in Chennai:

"Sex workers will maximum [/mostly/] be alcoholics. They say that only when they are drunk; they can do the work. We don't know whether this tablet can be taken if drunk? Suppose if it can be used even in drunken [state], then again taking it regularly will be difficult because if they take alcohol, they forget to take tablet regularly... These people [/alcoholics/] are not taking tablets regularly even for diabetes" [Urban BBSW, 05-10-30, Chennai, TN].

SBSW from Chennai pointed out towards the habit of consuming alcohol and raised apprehensions about interactions between alcohol and oral PrEP: 
"Most of the people in the field [/sex work/], they are having the habit of alcohol consumption. I have a doubt! While using this tablet, how it works at the time of alcohol intake?'[Urban SBSW, 05-10-21, Chennai, TN].

Alcohol was professed as part of their lifestyle. An SBSW from Pune described how she ends up having alcohol in her occupation and that she would be anxious about interaction with PrEP:

"Then my partner, if we have gone outside then what he does, at very first he brings beer, biryani [/a ricebased spicy and savory food/J, etc. If with him, then now... after taking the tablet, if I took a beer with him, then, will I not have trouble [/Taking beer with PrEP tablet: Would adverse effect develop? /]" [Urban

\section{SBSW, 01-10-60, Pune, MH]}

In an FGD at Chennai, an FSW also wanted to know about interactions with recreational drugs. One of the FSWs in the FGD also logically pointed out that because daily alcohol or drug intake was the compulsive need of their profession, taking daily oral PrEP will be a challenge:

"At the same time, I don't know whether those who are addicted to drugs they might take or not [/take oral PrEP/]. They feel that they would not be able to work if they are 'not intoxicated'[FSW-8].

A complete conviction seemed to emerge that this tablet should not be taken with alcohol or any other substance as the FGD participants from Pune put forth this argument:

"If having taken the tablet, then we should not chew Gutkha [/chewable tobacco/], should not drink [alcohol] and I have seen that with my eyes" [FSW-4].

Alcohol and drug use were the strong perceived reasons for not taking PrEP inadvertently: 'they will forget'; 'will be too intoxicated to remember' were commonly uttered phrases in the study. An SBSW from Pune told the reason for not wanting to take PrEP:

"Yes, hurdles... therefore we can't take [oral PrEP] daily and one thing about me is, I have the habit of drinking [alcohol]" [Urban SBSW, 01-10-60, Pune, MH].

The apprehensions surrounding PrEP medicine and alcohol was prevalent among FSWs. An SBSW from Pune narrated the sequelae of ending up with consuming alcohol for any or everything:

"As I drink alcohol, it means I cannot take that tablet [/Feels PrEP would interact with alcohol/]. Suppose I have white discharge then also I will not take that tablet; I will take it on the second day. But then, as I have severe white discharge; I will become angry and drink one quarter [/of alcohol/]. Woman [/FSW/] has such [habit] and [only] 5 out of 100 women [/FSWs/] are there who do not drink!'[Urban SBSW, 01-10-

\section{3, Pune, MH].}

Thus, fear of the adverse effect of oral PrEP in the context of their lifestyle was a major concern. 
Psychological barriers: A brief attention span and sustaining interest emerged as a unique challenge among FSWs. The fluctuating mood is aptly reflected in the phrase they used: 'anything routine is boring'. They do not want a repetition of any nature in their life and therefore, taking medicine every day is 'boring' for them.

"Will get bored. [People] will say, on daily basis-everyday what? Tablet-tablet! [/angry expression/]. Injection, if taken once, then there will be no tension up to one-one and a half year" [Urban SBSW, 01-1056, Pune, MH].

'Boring' was the state of mind that was constantly voiced by other stakeholders as something repetitive which is not acceptable. To prevent boredom with oral PrEP, other suggestions were that of intermittent dose or coitus dependent regimen that will not bind them to daily regimen:

"I have to eat [/take PrEP/] regularly, then people will be bored. This means they will miss out or become bored. So is it anything like this in that tablets, that the tablets will take only while during sex or like today suppose I want to do or want after one or two hours.... So instead of taking regularly, if the tablets [/PrEP/] will come like before the sex only, maybe half an hour, before 15-20 minutes should be taken then. There will not be any problem at all in that'[Key informant_Urban bisexual man, 01-30-11, Pune, MH].

Typological Barriers. The condition for non-use of oral PrEP would also depend on the typologies of the FSWs. Over half of the BBSWs (68\%) and almost a quarter of SBSWs (33\%) cautioned that there would be differential adherence to oral PrEP among FSWs belonging to various typologies. The situations and context are different for different typologies of FSWs. For example, a home-based sex worker would have probably no privacy to continue with the oral PrEP while FSWs, who were on daily-wages, would not be able to afford it daily because of cost as well as disrupted meal timings:

"Taking it daily in a family will not be easy. There is a possibility to forget due to situations like quarrels, any problem, and any work tension. If we become tense due to any problem, we might forget and then later worry about it" [Urban BBSW, 05-10-27, Chennai, TN].

"... majority of people are on daily wages, so it is difficult to take the tablet [/PrEP/] regularly" [Rural SBSW, 06-10-43, Vellore, TN].

Affordability emerged as a major challenge for adherence cited in case of adherence problems in treatment related to Non-Communicable Diseases and even prevention medicines. Majority of the FSWs demanded free or subsidized oral PrEP:

"It will be good if the government provides some concession or else free of cost on medicine for the people like us [/FSWs/] so that it will be very much helpful to take regularly" [Rural BBSW, 06-10-44, Vellore, TN].

Facilitators of adherence 
Adherence facilitators were very specific to the profession of FSWs. Their work required long-acting PrEP. Their work also required physical beauty/ attributes that they perceived that made them more attractive. Following two facilitators of adherence emerged: 1) Long-acting PrEP, 2) Monitoring

Long-acting PrEP: This emerged as the only facilitator for adherence from rural as well as urban FSWs:

"It's good if the tablet comes for 3 months once or twice but it should not be [/meant to be/] taken daily" [Rural SBSW, 06-10-43, Vellore, TN].

"The tablet [/PrEP/] can be made such that it can be taken weekly once or monthly once instead of daily. It will be better for people to take it consistently' [Urban SBSW, 05-10-21, Chennai, TN].

"Once we take for 2-4 months, if it works it is ok, or for once it works for one day it is ok.' 'UUrban SBSW, 0310-31, Belagavi, KT]

"It will be good if the tablet [/PrEP/] can be taken once in six months or yearly once, I feel difficult to use it daily'[Urban FGD, FSW-2, Chennai, TN].

An FSW took the example of national guidelines for HIV testing for key populations to justify a longacting PrEP as follows:

"Hmm! how our test [/HIV testing/] happens monthly or two months or three monthly? The same way women should get an injection like that ... so then she won't get bored" [Urban SBSW, 01-10-13, Pune, MH].

In the context of adherence, the FSWs put forth their fear of missing dose and the consequent repercussions of acquiring HIV. They tried to extrapolate from their past experiences with contraceptive pills where missed doses had led to conception. 'What happens if a dose is missed in case of unprotected sex'? This question was raised in several contexts particularly when these women were not confident of optimal adherence. A need to understand the impact of 'missed' dosage emerged as responded by the FSW in Chennai:

"If we see the contraceptive pills, we are taking it continuously for 30 days. If we leave it even for one day, we may conceive. But, for this [/oral PrEP/], if we take this continuously and leave it for one day, we should not get any effects [/HIV/]. If it is like that, then it is OK, or else it is difficult to take it continuously'[Urban BBSW, 05-10-27, Chennai, TN].

Monitoring: FSWs emphasized that community is error-prone when it comes to behaviors, especially health behaviors. Hence a simple logic was posed by them- 'no one gets punished for having a delay in meals'and similar principle should apply to oral PrEP. Concern for adherence followed logical exploration about the monitoring needs. FSWs suggested the role of NGOs in retention and adherence to overcome the problem of missing dose:

"Similarly they may not miss the tablet. Eventually, someone must follow them from the NGO to [/remind them to/] take the tablet regularly'[Rural SBSW, 06-10-43, Vellore, TN].

Page 15/26 


\section{Discussion}

FSWs were able to dwell upon the risk and uncertainties of their situation and use of oral PrEP. They discussed the effective evaluation of many alternatives and thus demand for oral PrEP became evident among FSWs both in the rural and urban settings in India. The fear of 'rape' was prevalent across urbanrural setting and as well as among street-based or brothel-based FSW. Brothel keepers were empathetic to the vulnerabilities in terms of coercion and rape of FSWs. The role of brothels in empowering sex workers in India have been well documented (21). Therefore, the empathy of brothel keepers and the trust of FSWs for them in this study can be utilized by involving brothels in oral PrEP rollout. They can play a vital role in ensuring acceptance and optimal adherence.

The need for oral PrEP emerges from reported vulnerabilities of coercive sex, hitherto, not discussed much in commercial sex work settings. Positive prevention or male condom usage, in either case, the control lies with the 'index case/ man' rather than the 'woman' who is more at the risk of acquiring HIV infection than the man. Thus, women who sell sex, might need self-controlled new HIV prevention options to protect themselves. A modeling study at Kenya and Ukraine estimates that a reduction of approximately $25 \%$ in HIV infections among sex workers may be achieved when physical or sexual violence is reduced (22). The ease and independence of the use of oral PrEP are helpful during situations of violence and coercion. FSWs from both the rural and urban settings professed their inability to negotiate male condom use. An alternative to male condoms was a demand among the FSWs. Our study shows that oral PrEP could be more empowering than condoms as oral PrEP needs to be 'swallowed' only. Hence, oral PrEP could be an important tool of prevention for this key vulnerable population.

Although the FSWs have responded to the HIV prevention program in India (23-26), yet in this study, the concept of prevention seemed to be difficult to comprehend, accept, practice and sustain. Our study found unique opportunities and challenges around PrEP usage among these different groups of FSWs. The compulsion to consume alcohol in their profession made FSWs realize their vulnerability. In an inebriated state of mind, FSWs acknowledged that they could not think about their protection. In that situation, 'a pill' rather than any other prevention product seemed to be a highly acceptable prevention option empowering them to be prepared before they became intoxicated and vulnerable. The locus of control was aptly perceived to lie with the FSW and nothing was easier than swallowing a pill; hence a strong willingness to use oral PrEP as HIV prevention option among FSWs emerges from this study. Unlike other studies where long acting injectable PrEP was preferred (27), in our study, FSW wanted 'pill' on which they could have control unlike injectables which were perceived as health care provider driven. FSWs from both urban and rural settings voiced their fear of stigma and tablet was an acceptable formulation both to circumvent stigma, and maintain privacy. FSWs reported being more comfortable with NGO/ CBOs functionaries as compared to clinicians with whom they felt awkward or stigmatized. This addresses the problem of the potential burden on HCPs because community-based implementers were reportedly more acceptable. Along with brothel keepers, grassroots level workers from the community (Anganwadi workers) were the acceptable implementer option and they can share the task 
during oral PrEP implementation. This can bring about better community acceptance and might ensure adherence to PrEP.

Oral PrEP was perceived as a tool for economic enhancement among the FSWs. Other studies have also shown that FSW might forgo condom use for earning more (28). However, risk compensation remains a concern and condoms need to be used for pregnancy and sexually transmitted infections prevention. FSWs should be reminded strongly that oral PrEP would not protect them from other STIs or pregnancy. In mathematical modeling, it has already been shown that the impact of PrEP may be strongly diminished or even reversed by behavioral disinhibition, especially in scenarios with low coverage and low effectiveness (29). Thus, oral PrEP as an addition and not as substitution should be offered in the Indian setting. Roll out with a strong statutory warning that it might protect against HIV but additional prevention options would be required for STI/ pregnancy prevention should be implemented. Roll out of oral PrEP would require policymakers to recommend such legislations.

Issues of non-acceptability of oral PrEP among regular condom users also emerged which can be solved by bringing oral PrEP as an addition to the prevention option without undermining existing prevention programs. However, it is also to be noted that switching over from condom to PrEP and vice versa might not reverse the effects of PrEP even if condom use reduces by $50 \%$ among sex workers (30).

Poverty breeds compromises and in this study, the examples of compromises were rampant use of alcohol and substance along with the addition of condom less sex among FSWs. This has also been reported in another study conducted in India (31). Zhang et al (2013) stated that the compulsion to consume alcohol is a typical vulnerability of low paid sex workers and in their study, they were generally street-based sex workers (32). By this definition, in our study, rural FSWs draw attention to their lowest level of safety and high episodes of violence and coercion ranking them as the lowest in the hierarchy of sex workers. Rural FSWs also pointed out being daily wage earner and related this to their inability to adhere to a daily dosage of PrEP. It not only indicates affordability issues but also adherence could be a concern for a daily regimen. The interventions would be a challenge in a rural setting but an urgent need for prevention options is critical. The rural FSWs wanted the involvement of government-run ART centers for dispensing and follow-up which was very different from what urban FSWs wanted.

Concerns for side effects have been voiced worldwide (33). The systemic side effects could be a deterrent to oral PrEP usage among FSWs but might be tolerated with medical intervention and counseling especially the self-limiting ones (34). The FSWs in this study appeared very cautious in sharing their preference for oral PrEP. Evidence of intrinsic fear of side effects emerged repeatedly; almost all of them put forth the condition that the product should be tested first. A need for a demonstration project also emerged as FSWs wanted to 'see and use' the product before forming any concrete opinion on the optimal usage of oral PrEP. Several side effects which were the symptoms of Reproductive Tract Infections (RTIs) were openly associated with any medical product being used by them. The demonstration project would also address the challenge of such mythical side effects that were cited by 
FSWs. Counseling about possible side effects and symptoms of RTIs/STIs should be a part of a PrEP rollout program.

Three of the five influencers stated by Parkin (17) tend to influence the decision to take daily oral PrEP. The personality of not liking repetitive things (Viz. daily regimen), personal values such as faith upon regular partner and having alcohol as social and occupational norms were constraints emerging for the use of daily oral PrEP among this population. The personal value also emerged, when the apprehension about side effects on their physical attributes that are especially valued in the profession of this key population emerged. The side effects on the physical attribute i.e. the beauty and reproductive health were not at all acceptable in place of any kind of protection/ prevention. The concern for the protection of beauty and reproductive parts provides a key message to the researchers, drug developers and pharmaceuticals that they must focus their attention on the side effects especially on body fat changes, side effects to the uterus or other reproductive parts. The occurrence of such side effects would spell the failure of oral PrEP rollout among the FSW population. FSWs were also concerned about the interaction between alcohol and oral PrEP. Messages clarifying that there would not be oral PrEP-alcohol interaction was a demand that needs to be fulfilled if optimal adherence is expected. Alongside it is also important to emphasize the messages that there would be an effect of alcohol on their behaviors that would have an impact on adherence.

Adherence remains a strong prescription both for HIV prevention and treatment. In PrEP trials, adherence emerged as one of the critical factors influencing effectiveness (35). Adhering to the daily regimen was one of the most difficult problems stated by the FSWs in this study; especially in the wake of their routine alcohol/ recreational drug use. Alcohol and recreation as barriers to adherence to PrEP were reported elsewhere also (28). Their expected subsequent state of intoxication made them hesitate about regular usage of oral PrEP. Strong adherence counseling and retention strategies are required.

As observed in large clinical trials (36), stigma about ART was a barrier for both acceptance and adherence to oral PrEP. ART, which is associated with HIV treatment, led to the fear of stigma and being labeled as HIV infected. In other studies too, regular pill taking of oral PrEP, alcohol consumption and irregular lifestyles and fear of being perceived as HIV positive has been documented in other studies too $(28,34)$. Since oral PrEP would be an ART, it posed a barrier to acceptance and adherence to oral PrEP. FSWs' concern for adherence emerged from their experience with family planning. The expectation was that oral PrEP default should be more forgiving i.e. one event of non-adherence should not lead to HIV acquisition. Hence emerges the need for products with longer half-life to achieve a target steady-state level more quickly (37) especially in the populations where adherence is bound to be sub-optimal. FSWs' concerns regarding adherence of daily dosage is a critical issue. The unique feature of the FSW community appears to be their affinity for continuous change. They feared boredom and anything 'routine' was boring. 'Boredom' is known to breed sensation-seeking behavior (38) which perhaps explains an FSWs' risky and sometimes pompous lifestyle. Easily bored people are discontent, at higher risk of depression, anxiety, drug addiction, alcoholism, etc. Therefore, in due course, this trait is bound to influence acceptability and adherence to prevention products in this population. Repetition not only made 
them wary of the long term side effects of oral PrEP, but they also felt that it compromised their ability to adhere to the daily regimen of oral PrEP. Hence, there was resistance to 'daily' PrEP. Again, long-acting HIV prevention regimens need to be pursued.

Stigma encompasses every aspect of FSWs' life. To fight stigma, FSWs keep struggling and a critical situation is when she voluntarily refuses to use prevention methods. The dynamics of intimate relationships among FSWs and their regular partners show the non-efficacy of FSWs to use HIV prevention options. In a study among sex workers in Mexico, Robertson et al suggested modifying the HIV risk framework which only focuses on constructs of disease and risk (39). As in this Mexican study, FSWs in our study also ascribed a different meaning to their relationship with regular partners which needs to be considered for implementing oral PrEP rollout among FSWs in India. Psychological empowerment might overcome the adverse effects of stigma and exclusion among FSWs (40).

In line with another study (13), FSWs in this study also pointed towards stigmatizing the health care system. Easy accessibility at pharmacies or involvement of CBOs or grass root workers from health system for dispensing was preferred and an aversion for health care provider mediated oral PrEP emerged. Oral PrEP seems to be more empowering because of the non-intervention of health care providers, unlike Kenyan FSWs who preferred injectable PrEP (28). Our study emphasizes a need to clarify to the FSW population that initiation of oral PrEP and subsequent monitoring of side effects and adherence by skilled medical staff is critical for at least the initial few months. The expectation that oral PrEP will be freely available needs to be thwarted as early as possible. A peer-led approach for dispensing of PrEP in conjunction with formal health care settings is recommended. The ART retention model of peer involvement existing in India can be adapted for the oral PrEP program. The role of brothel owners is already discussed.

The social marketing model for oral PrEP delivery emerges. In rural areas confidentiality was a larger concern and preference was for delivery through ART centers while in urban areas preference was for NGOs. This issue of dispensing needs to be explored. According to a UNAIDS meeting report (2014), the introduction of PrEP to sex workers should be integrated within the secure provision of comprehensive sexual health care, including HIV treatment. It also recommended widespread monitored access to PrEP is necessary to reduce informal and unregulated use (41).

An optimism emerged around PrEP but it is to be noted that FSWs have been placed in a wide bracket of people at risk of HIV. FSWs questioned the need for prevention in the absence of disease or symptoms indicating their right to exercise to make sexual health decisions on their own. This is also an expectation to define 'substantial risk for oral PrEP indication' like it is defined in case of Men having Sex with Men and transgender. Their suggestions that it should be 'used first' before they can give their preference, perhaps point out the gap such as lack of evidence of how would oral PrEP specifically impacts them in their own socio-cultural and occupational setting in the long run. To improve effectiveness in real-world settings, more research is needed on feasibility of using PrEP in countries like India and the contextual factors surrounding women's personal experiences with PrEP (42). It does not exonerate the country from 
conducting a demonstration project among female sex workers. In the larger interest, a higher-order structural intervention is needed to make community members realize the goal of public health which is through prevention.

\section{Study limitations}

The limitations is that data is not very current; however, since India is still preparing to roll out PrEP program, this study informs the program.

\section{Conclusion}

Several issues emerge for oral PrEP rollout among FSWs in India. At the structural level, violence emerges as a strong inhibitor of an FSW's ability to protect herself. HIV prevention program in India needs to implement strategies to address violence against FSWs. FSWs are treated as non-independent entities: 1) they can neither refuse clients nor regular partners, 2) they are in the broad category of the key population but have their unique ramifications of risks. Oral PrEP is hoped to provide them protection in complete privacy. Although, they do not want HCP initiated PrEP yet, initially HCPs need to be involved. It would require planned advocacy, explaining the need for HCP to assess the safety monitoring of oral PrEP. High hopes about oral PrEP as a replacement for condom reiterates the need to offer it as addition and not substitution. A multisite demonstration project to assess the feasibility of PrEP among FSWs at the community level and standard health care settings is needed before its rollout. The effectiveness of PrEP is directly proportional to adherence and adherence, as expected, remained a prominent challenge owing to alcohol, boredom, and fear of side effects. FSWs would need to be assured about the safety of physical attributes and the reproductive system. Product developers should develop innovative longacting or coitus dependent products that would address adherence issues. Participatory efforts are essential for PrEP to roll out into an effective and available intervention among FSWs in India. To address the stigma associated with HIV and ART, the introductory programs for PrEP roll-out must be designed carefully along with the need to develop the strategies for the availability of products without breaching confidentiality. The fear of being labeled as HIV positive because they are taking PrEP provides a clue to the program managers on positioning oral PrEP very clearly as prevention product; a product completely different from ARV. Owing to the established trust among FSWs, NGOs/ CBOs working with FSWs, grassroot level workers and brothel keepers may share the task and make the PrEP rollout program acceptable and effective. Peer-led oral PrEP dispensing integrated with formal health care sectors could spell the success of the oral PrEP roll-out program among the FSW population. FSWs in rural and urban settings have varying issues of stigma which culminates into wanting or not wanting to involve HCPs respectively. Engaging brothel keepers in the oral PrEP roll-out program would be useful as they are the only support system existing for FSWs who understand their situational vulnerabilities. To formulate a PrEP package in India, the broader HIV/AIDS-related national policies and programs may need to evolve to increase the availability, acceptability, and utilization of oral PrEP among the most vulnerable populations.

\section{Abbreviations}


PrEP: Pre-Exposure Prophylaxis

FSW: Female Sex Workers

BBSW: Brothel Based Sex Worker

SBSW: Street Based Sex Worker

NGO: Non-governmental Organization

CBO: Community Based Organization

\section{Declarations}

Ethics approval and consent to participate: The study was approved by the Institutional Ethics Committee of ICMR- National AIDS Research Institute (Pune, Maharashtra), ICMR-National Institute of Epidemiology (Chennai, Tamil Nadu), and Jawaharlal Nehru Medical College (Belagavi, Karnataka). Written informed consent was obtained from all the study participants prior to their participation in the study.

Consent to publish: Not Applicable

Availability of data and material: Data will be available at the NARI's centralized data repository system and is available on request to director@nariindia.org.

Competing interests: The authors of this manuscript declare that they have no competing interests.

Funding: The funding support was received from the Indian Council of Medical Research, New Delhi (Letter no. 5/7/703/09-RHN). The funders had no role in study design, data collection and analysis, decision to publish, or preparation of the manuscript.

Authors' Contribution: SS, NC, TS and MA planned and implement the study. SS, TS, MA, AB and AV developed the tools; $A B$ and $A V$ were involved in data collection and date entry; SS and AV were involved in data analysis; SS, AV, SPS, SD, AB, MA, TS and NC were involved in manuscript preparation. All authors have read and approved the manuscript.

Acknowledgments: We acknowledge Indian Council of Medical Research for the financial support (Letter no. 5/7/703/09-RHN) for this study. We are immensely thankful to the study respondents for sharing their views and sensitive information with us. We would like to thank our staff Mr. Mufid Baig, Mr. Suryakant Borade, Mr. Rais Patel, Mr. Vinod Bhalerao, Mrs. Ratnaprabha Potdar and Ms. Sujata Kamble for their constant support in recruitment and data collection. We thank Dr. Ashwani Angadi, Mrs. K. Preethi and Mrs. Poonguzhali for their continuous support in recruitment, data collection and processing at Chennai and Belagavi site. We also thank Mr. Basit Momin, Ms. Sujata Kamble, Mr. Anand Kumar and Mr. Basavraj $\mathrm{G}$ for entering the data at sites. 


\section{References}

1. NACO. India's Voice against AIDS. In: Control DoA, editor. New Delhi: National AIDS Control Organisation, Ministry of Health and Family Welfare, Government of India. 2013.

2. NACO. Targeted Interventions under NACP III: Core High Risk Groups. Volume I. National AIDS Control Organisation, Ministry of Health \& Family Welfare, Government of India. [Internet]. 2007 [cited 2020 Mar 21]. Available from: http://naco.gov.in/sites/default/files/NACP-III.pdf

3. NACO. National AIDS Control Organization. Annual Report. In: Control DoA, editor. New Delhi: Ministry of Health and Family Welfare, Government of India; 2014-15. 2015.

4. Cáceres CF, Koechlin F, Goicochea P, Sow P-S, O’Reilly KR, Mayer KH, et al. The promises and challenges of pre-exposure prophylaxis as part of the emerging paradigm of combination HIV prevention. Journal of the International AIDS Society [Internet]. $2015 \mathrm{Jul}$ [cited $2020 \mathrm{Mar}$ 22];18:19949. Available from: http://doi.wiley.com/10.7448/IAS.18.4.19949

5. NACO. Narrative country progress report of India: Global AIDS Response Progress Reporting 2015. New Delhi: National AIDS Control Organisation. Contract No.: GOI/NACO/SIM/GR-RPT/200415. 2015.

6. Abdool Karim Q, Abdool Karim SS, Frohlich JA, Grobler AC, Baxter C, Mansoor LE, et al. Effectiveness and safety of tenofovir gel, an antiretroviral microbicide, for the prevention of HIV infection in women. Science. 2010 Sep 3;329(5996):1168-74.

7. Baeten JM, Donnell D, Ndase P, Mugo NR, Campbell JD, Wangisi J, et al. Antiretroviral prophylaxis for HIV prevention in heterosexual men and women. N Engl J Med. 2012 Aug 2;367(5):399-410.

8. Thigpen MC, Kebaabetswe PM, Paxton LA, Smith DK, Rose CE, Segolodi TM, et al. Antiretroviral preexposure prophylaxis for heterosexual HIV transmission in Botswana. N Engl J Med. 2012 Aug 2;367(5):423-34.

9. Van Damme L, Corneli A, Ahmed K, Agot K, Lombaard J, Kapiga S, et al. Preexposure prophylaxis for HIV infection among African women. N Engl J Med. 2012 Aug 2;367(5):411-22.

10. Fonner VA, Dalglish SL, Kennedy CE, Baggaley R, O'Reilly KR, Koechlin FM, et al. Effectiveness and safety of oral HIV preexposure prophylaxis for all populations. AIDS. 2016 31;30(12):1973-83.

11. Cohen MS, Baden LR. Preexposure prophylaxis for HIV-where do we go from here? N Engl J Med. 2012 Aug 2;367(5):459-61.

12. Baeten J, Celum C. Systemic and topical drugs for the prevention of HIV infection: antiretroviral preexposure prophylaxis. Annu Rev Med. 2013;64:219-32.

13. Cowan FM, Delany-Moretlwe S. Promise and pitfalls of pre-exposure prophylaxis for female sex workers. Curr Opin HIV AIDS. 2016 Jan;11(1):27-34.

14. Auerbach JD, Hoppe TA. Beyond "getting drugs into bodies": social science perspectives on preexposure prophylaxis for HIV. J Int AIDS Soc. 2015;18(4 Suppl 3):19983.

15. Reza-Paul S, Lazarus L, Jana S, Ray P, Mugo N, Ngure K, et al. Community Inclusion in PrEP Demonstration Projects: Lessons for Scaling Up. Gates Open Res [Internet]. 2019 Dec 17 [cited 2020 
Mar 21];3:1504. Available from: https://gatesopenresearch.org/articles/3-1504/v2

16. Bandura A. Social foundations of thought and action: a social cognitive theory. Englewood Cliffs, N.J: Prentice-Hall; 1986. 617 p. (Prentice-Hall series in social learning theory).

17. Parkin J. Organizational decision making and the project manager. International Journal of Project Management [Internet]. 1996 Oct [cited 2020 Mar 21];14(5):257-63. Available from:

https://linkinghub.elsevier.com/retrieve/pii/026378639684508X

18. Ryan GW, Bernard HR. Techniques to Identify Themes. Field Methods [Internet]. 2003 Feb [cited 2020 Mar 22];15(1):85-109. Available from:

http://journals.sagepub.com/doi/10.1177/1525822X02239569

19. Strauss AL, Corbin JM. Basics of qualitative research: grounded theory procedures and techniques. 3. printing. Newbury Park, Calif.: Sage; 1991. 270 p.

20. Glaser BG, Strauss AL. The purpose and credibility of qualitative research. Nurs Res. 1966;15(1):5661.

21. Ghose T, Swendeman DT, George SM. The role of brothels in reducing HIV risk in Sonagachi, India. Qual Health Res. 2011 May;21(5):587-600.

22. Decker MR, Wirtz AL, Pretorius C, Sherman SG, Sweat MD, Baral SD, et al. Estimating the impact of reducing violence against female sex workers on HIV epidemics in Kenya and Ukraine: a policy modeling exercise. Am J Reprod Immunol. 2013 Feb;69 Suppl 1:122-32.

23. Armstrong G, Medhi GK, Kermode M, Mahanta J, Goswami P, Paranjape R. Exposure to HIV prevention programmes associated with improved condom use and uptake of HIV testing by female sex workers in Nagaland, Northeast India. BMC Public Health [Internet]. 2013 Dec [cited 2020 Mar 22];13(1):476. Available from: http://bmcpublichealth.biomedcentral.com/articles/10.1186/14712458-13-476

24. Bhattacharjee P, Isac S, McClarty LM, Mohan HL, Maddur S, Jagannath SB, et al. Strategies for reducing police arrest in the context of an HIV prevention programme for female sex workers: evidence from structural interventions in Karnataka, South India. J Int AIDS Soc. 2016;19(4 Suppl 3):20856.

25. Nagarajan K, Sahay S, Mainkar MK, Deshpande S, Ramesh S, Paranjape RS. Female sex worker's participation in the community mobilization process: two distinct forms of participations and associated contextual factors. BMC Public Health. 2014 Dec 24;14:1323.

26. Vejella S, Patel SK, Saggurti N, Prabhakar P. Community Collectivization and Consistent Condom Use Among Female Sex Workers in Southern India: Evidence from Two Rounds of Behavioral Tracking Surveys. AIDS Behav. 2016 Apr;20(4):776-87.

27. Luecke EH, Cheng H, Woeber K, Nakyanzi T, Mudekunye-Mahaka IC, van der Straten A, et al. Stated product formulation preferences for HIV pre-exposure prophylaxis among women in the VOICE-D (MTN-003D) study. J Int AIDS Soc. 2016;19(1):20875.

28. Mack N, Evens EM, Tolley EE, Brelsford K, Mackenzie C, Milford C, et al. The importance of choice in the rollout of ARV-based prevention to user groups in Kenya and South Africa: a qualitative study. $J$ 
Int AIDS Soc. 2014;17(3 Suppl 2):19157.

29. Vissers DCJ, Voeten HACM, Nagelkerke NJD, Habbema JDF, de Vlas SJ. The impact of pre-exposure prophylaxis (PrEP) on HIV epidemics in Africa and India: a simulation study. PLoS ONE. 2008 May 7;3(5):e2077.

30. Mitchell KM, Prudden HJ, Washington R, Isac S, Rajaram SP, Foss AM, et al. Potential impact of preexposure prophylaxis for female sex workers and men who have sex with men in Bangalore, India: a mathematical modelling study. J Int AIDS Soc. 2016;19(1):20942.

31. Panchanadeswaran S, Johnson SC, Sivaram S, Srikrishnan AK, Latkin C, Bentley ME, et al. Intimate partner violence is as important as client violence in increasing street-based female sex workers' vulnerability to HIV in India. Int J Drug Policy. 2008 Apr;19(2):106-12.

32. Zhang C, Li X, Hong Y, Zhou Y, Liu W, Stanton B. Unprotected sex with their clients among low-paying female sex workers in southwest China. AIDS Care. 2013;25(4):503-6.

33. Ye L, Wei S, Zou Y, Yang X, Abdullah AS, Zhong X, et al. HIV pre-exposure prophylaxis interest among female sex workers in Guangxi, China. PLoS ONE. 2014;9(1):e86200.

34. Van der Elst EM, Mbogua J, Operario D, Mutua G, Kuo C, Mugo P, et al. High acceptability of HIV preexposure prophylaxis but challenges in adherence and use: qualitative insights from a phase I trial of intermittent and daily PrEP in at-risk populations in Kenya. AIDS Behav. 2012 Jul;17(6):2162-72.

35. van der Straten A, Van Damme L, Haberer JE, Bangsberg DR. Unraveling the divergent results of preexposure prophylaxis trials for HIV prevention. AIDS. 2012 Apr 24;26(7):F13-19.

36. van der Straten A, Stadler J, Luecke E, Laborde N, Hartmann M, Montgomery ET. Perspectives on use of oral and vaginal antiretrovirals for HIV prevention: the VOICE-C qualitative study in Johannesburg, South Africa. Journal of the International AIDS Society [Internet]. 2014 Sep 8 [cited 2020 Mar 22];17(3(Suppl 2)). Available from: http://doi.wiley.com/10.7448/IAS.17.3.19146

37. Teuscher N. The Roundtable: Our Thoughts About Model Based Drug Development: Understanding steady state pharmacokinetics. [Internet]. New Jersey, USA: Certara; 2013. Available from: https://www.certara.com/2013/11/25/understanding-steady-state-pharmacokinetics/

38. Gosline A. Bored to Death: Chronically Bored People Exhibit Higher Risk-Taking Behavior [Internet]. Scientific American. 2007 [cited 2020 Mar 22]. Available from: https://www.scientificamerican.com/article/the-science-of-boredom/

39. Robertson AM, Syvertsen JL, Martinez G, Rangel MG, Palinkas LA, Stockman JK, et al. Acceptability of vaginal microbicides among female sex workers and their intimate male partners in two MexicoUS border cities: a mixed methods analysis. Glob Public Health. 2013;8(5):619-33.

40. Loury GC. Social Exclusion and Ethnic Groups: The Challenge to Economics. 1999;41.

41. UNAIDS. Sex Workers' Hopes and Fears for HIV Pre-Exposure Prophylaxis: Recommendations from a UNAIDS Consultation Meeting. 11-12 November, Johannesburg. [Internet]. AVAC. 2014 [cited 2020 Mar 22]. Available from: https://www.avac.org/resource/sex-workers-hopes-and-fears-hiv-preexposure-prophylaxis-recommendations-unaids 
42. Strathdee SA, Wechsberg WM, Kerrigan DL, Patterson TL. HIV prevention among women in low- and middle-income countries: intervening upon contexts of heightened HIV risk. Annu Rev Public Health. 2013;34:301-16.

\section{Figures}

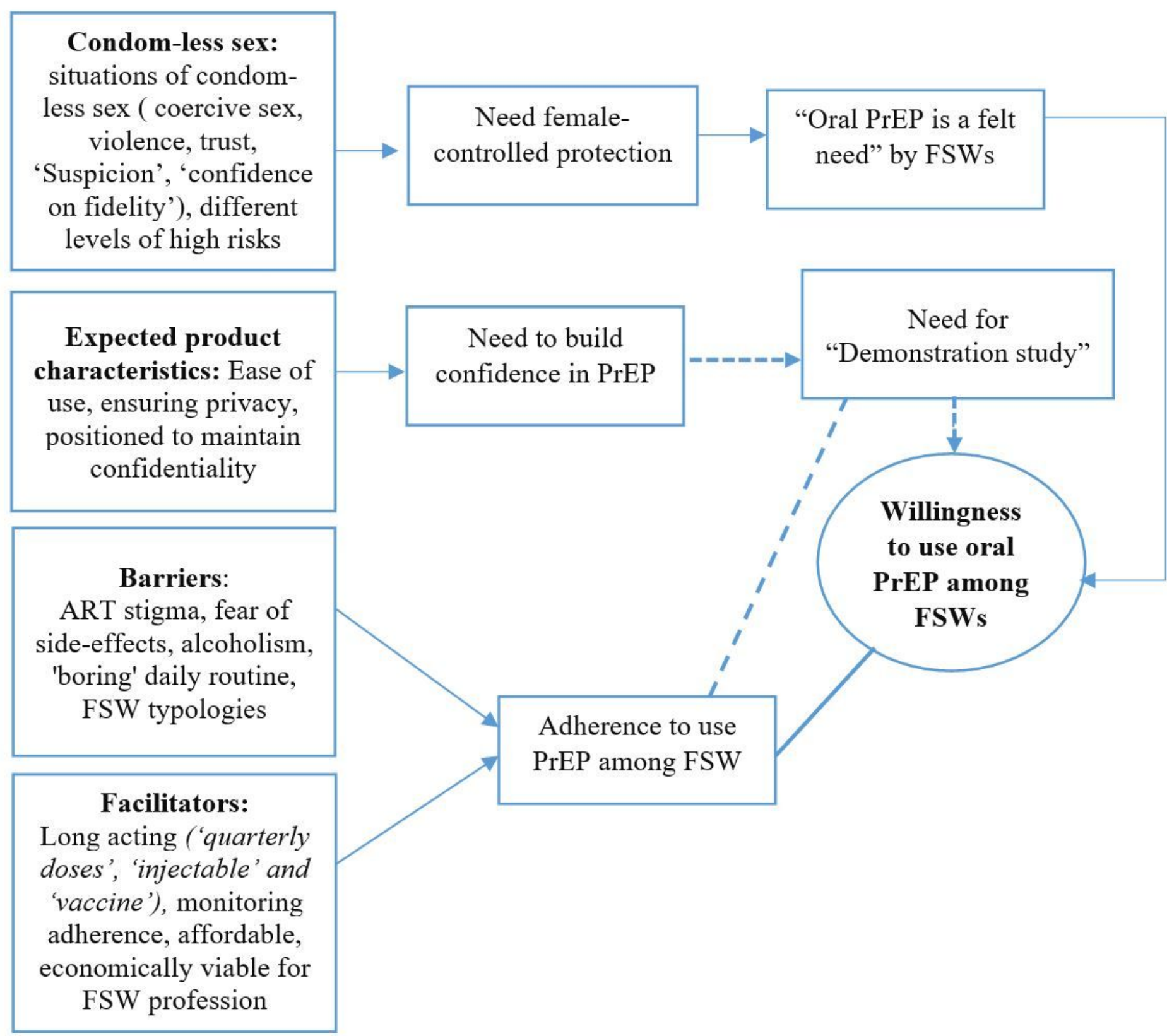

\section{Figure 1}

Analytical framework for emerging needs for oral PrEP use among FSWs The need for PrEP emerged because FSWs were constantly at risk even if they were aware of condom and wanted to use condom. Vulnerability narrated by FSWs brought forth the 'need' for oral PrEP. Demand for demonstration study emerged as influencer of usage but there were other facilitators that could influence usage independent 
of demonstration studies. Long action products would help in future adherence. The dotted lines indicate desire for demonstration project or need for contextual evidence. 